\title{
FOXQ1 is overexpressed in laryngeal carcinoma and affects cell growth, cell cycle progression and cell invasion
}

\author{
JIE ZHANG $^{1}$, WEI LI ${ }^{1}$, SONG DAI $^{2}$, XUHUI TAI ${ }^{2}$, JIANPING JIA $^{2}$ and XING GUO ${ }^{1}$ \\ ${ }^{1}$ Department of Otolaryngology, The First Affiliated Hospital of China Medical University, Shenyang, Liaoning 110001; \\ ${ }^{2}$ Department of Otolaryngology, The 463 Hospital of PLA, Shenyang, Liaoning 110007, P.R. China
}

Received September 8, 2014; Accepted June 11, 2015

DOI: $10.3892 / \mathrm{ol} .2015 .3530$

\begin{abstract}
Forkhead box Q1 (FOXQ1) is a forkhead transcription factor that is involved in numerous biological processes and has been shown to participate in tumorigenesis. However, the clinical significance of the expression of this protein in laryngeal carcinoma, and the mechanisms underlying its regulation in this disease remain unclear. The aim of present study was to measure the expression of FOXQ1 in laryngeal carcinoma, and to examine its effect on tumorigenesis. In the present study, reverse transcription-quantitative polymerase chain reaction and western blotting were employed to measure FOXQ1 expression in laryngeal carcinoma tissue samples, small interfering RNA specific to FOXQ1, was transfected into Hep2 cells and its effect on cell proliferation, cell cycle progression and cell migration was examined, using a CCK-8 assay, flow cytometry and a transwell migration assay, respectively. The results showed overexpression of FOXQ1 mRNA and protein in laryngeal cancer tissue samples. Inhibition of FOXQ1 suppressed cell growth and invasion, and arrested cells in the G0/G1 phase. Overexpression of FOXQ1 is associated with the development of laryngeal carcinoma and may enhance tumorigenesis through its effects on cell proliferation, cell cycle progression and cell migration.
\end{abstract}

\section{Introduction}

Head and neck squamous cell carcinoma (HNSCC) is the eighth leading cause of cancer-related mortality worldwide (1). Laryngeal squamous cell carcinoma (LSCC), the second most common malignant neoplasm of the upper respiratory tract, is a subtype of HNSCC (2). Approximately 10,000 new cases of LSCC are diagnosed each year in the United States (3). In China, the incidence of LSCC is increasing, particularly in

Correspondence to: Professor Xing Guo, Department of Otolaryngology, The First Affiliated Hospital of China Medical University, 155 Nanjing North Street, Shenyang, Liaoning 110001, P.R. China

E-mail: 17957361@qq.com

Key words: laryngeal carcinoma, forkhead box Q1, proliferation, invasion the country's Northeast region (4). Significant predisposing factors to the development and progression of LSCC, include alcohol abuse and tobacco (5). Early stage LSCC may be effectively treated with surgery or radiotherapy (6). When diagnosed at an advanced stage, this disease usually requires a combination of treatment modalities. However, although such combined therapy has improved local control and overall quality of life, the local recurrence rate varies from $10-50 \%$, depending on tumor stage and the overall survival rate has not improved significantly over two decades $(7,8)$. Therefore, it is necessary to identify novel biomarkers for use in the diagnosis of LSCC. In addition, the study of the molecular mechanisms underlying the development of LSCC may improve treatment and increase survival for patients with this disease.

Forkhead box Q1 (FOXQ1, also termed HFH1) is a member of the forkhead transcription factor family (9), which is involved in a variety of biological processes, including epithelial differentiation (10), cell cycle progression (11), embryonic stem cell differentiation $(12)$, metabolism $(13,14)$ and carcinogenesis (15-17). As one of the first forkhead genes to be investigated, FOXQ1 has been demonstrated to be involved in metabolism, aging (18) and carcinogenesis (19). Overexpression of the FOXQ1 protein is associated with epithelial-mesenchymal transition (EMT) and a poor prognosis in certain types of cancer, such as non-small cell lung cancer (20) and breast cancer (21). However, little is known regarding FOXQ1 expression in LSCC and its involvement in the pathogenesis of this disease. The present study aimed to investigate the effect of FOXQ1 expression on the development and progression of LSCC, by measuring its expression in LSCC tissue samples, and investigating its effect on cell proliferation, cell cycle progression and cell migration.

\section{Materials and methods}

Patients and tissue samples. Thirty pairs of LSCC and corresponding adjacent normal tissues, used for reverse transcription-quantitative polymerase chain reaction (RT-qPCR) and western blotting, were collected from the Ear, Nose and Throat department of The 463 Hospital of PLA (Shenyang, China) between January 2009 and December 2014 , following receipt of written informed consent. Tissue samples were obtained from 24 males and 6 females (mean 
age, 64.72 years; range, $45-83$ years) and included 6 cases of stage I LSCC, 6 cases of stage II LSCC, 8 cases of stage III LSCC and 10 cases of stage IV LSCC. Tumors were staged according to the International Union Against Cancer TNM classification for malignant tumors (22). All tissue samples, including cancer tissues and matched adjacent normal tissues (typically removed from areas $4-15 \mathrm{~mm}$ from the tumors), were obtained during surgery. All specimens were frozen and stored at $-80^{\circ} \mathrm{C}$ prior to use. Approval for this study was obtained from the Ethics Committee of China Medical University (Shenyang, China).

Cell culture. The Hep2 human laryngeal carcinoma cell line, was obtained from the Shanghai Institute for Biochemistry, Chinese Academy of Sciences (Shanghai, China). Cells were cultured in RPMI-1640 (Gibco Life Technologies, Carlsbad, CA, USA), supplemented with $10 \%$ fetal bovine serum, $100 \mu \mathrm{g} / \mathrm{ml}$ penicillin and $100 \mu \mathrm{g} / \mathrm{ml}$ streptomycin (all obtained from GE Healthcare Life Sciences, Logan, UT, USA) in humidified $5 \% \mathrm{CO}_{2}$ at $37^{\circ} \mathrm{C}$. Trypsin solution (0.25\%; GE Healthcare Life Sciences) was used to detach cells from the culture flask.

Transient transfection with FOXQ1-specific small interfering RNA (siRNA). Three siRNAs targeting human FOXQ1 and a negative control siRNA (FOXQ1-NC), were designed and obtained from GenePharma Co., Ltd. (Shanghai, China). The siRNAs and FOXQ1-NC siRNA (FOXQ1-siRNA1, 5'-CGCGGACTTTGCACTTTGA-3'; FOXQ1-siRNA2, 5'-AGGGAACCTTTCCACACTA-3'; FOXQ1-siRNA3, 5'-CCATCAAACGTGCCTTAAA-3'; and FOXQ1-NC siRNA, 5'-TTCTCCGAACGTGTCACGT-3') were used to inhibit the expression of FOXQ1. Preliminary experiments indicated that FOXQ1-siRNA1 most effectively down-regulated FOXQ1 expression. This sequence was therefore selected for subsequent experiments. Hep2 cell were seeded in 6-well plates at a density of $0.5 \times 10^{6}$ cells/well. FOXQ1-siRNA, FOXQ1-NC and mock group (blank control \pm transfection reagent) were transfected into Hep2 cells using lipofectamine 2000 transfection reagent (Invitrogen Life Technologies, Carlsbad, CA, USA), according to the manufacturer's instructions. Following transfection for $72 \mathrm{~h}$, cells were collected for subsequent experiments.

RNA isolation and RT-qPCR. Total RNA was extracted using TRIzol ${ }^{\mathrm{TM}}$ reagent (Invitrogen Life Technologies) for analysis of FOXQ1 and GAPDH mRNA expression, according to the manufacturer's instructions. RNA was reverse transcribed, using the Reverse Transcription PCR kit with Oligo-dT primers and RT-qPCR was conducted, using SYBR-Premix Ex Taq ${ }^{\mathrm{TM}}$ (Takara Bio Inc., Shiga, Japan), according to the manufacturer's instructions. For detection of FOXQ1-mRNA expression, qPCR was performed under the following conditions: Denaturation at $95^{\circ} \mathrm{C}$ for $30 \mathrm{sec}$, followed by 40 cycles of amplification (annealing at $95^{\circ} \mathrm{C}$ for $5 \mathrm{sec}$ and elongation at $60^{\circ} \mathrm{C}$ for $30 \mathrm{sec}$ ). GAPDH was used to normalize FOXQ1-mRNA expression levels using the $2^{-\Delta \Delta \mathrm{Ct}}$ method. The following primers were used: Forward, 5'-ATTTCTTGCTATTGACCGATGC-3' and reverse, 5'-CCCAAGGAGACCACAGTTAGAG-3' for FOXQ1 and forward, 5'-GGAAGATGGTGATGGGATT-3' and reverse, 5'-GGATTTGGTCGTATTGGG-3' for GAPDH. All primers were purchased from Takara Bio, Inc.

Western blotting. Western blot analysis was performed according to standard procedures. In brief, protein was isolated from tissue samples or cells. Protein concentration was determined using a bicinchoninic acid Protein Assay kit (Pierce Biotechnology, Inc., Rockford, IL, USA). Proteins were fractionated using SDS-PAGE (Invitrogen Life Technologies) and transferred to PVDF membranes (Beyotime Institute of Biotechnology, Haimen, China). After blocking with 5\% milk in Tris-buffered saline with Tween-20 (TBST; Invitrogen Life Technologies), membranes were incubated with a polyclonal rabbit anti-human FOXQ1 antibody (cat. no. sc-134549; Santa Cruz Biotechnology, Inc., Dallas, TX, USA) at a 1:1,000 dilution over $3 \mathrm{~h}$. The membranes were then washed thrice with TBST, and incubated with horseradish peroxidase-conjugated polyclonal goat anti-rabbit (cat. no. KC-MM-095) or goat anti-mouse (cat. no. KC-MM-035) secondary antibodies (KangCheng, Shanghai, China) at a 1:2,000 dilution for $2 \mathrm{~h}$ at room temperature. The membranes were also stripped and blotted with a monoclonal mouse anti-human $\beta$-actin antibody (cat. no. A5316; Sigma-Aldrich, St. Louis, MO, USA) at a 1:1,000 dilution, as a loading control. Blots were developed with enhanced chemiluminescence and chemiluminescence detection film (Beyotime Institute of Biotechnology).

Cell proliferation assay. Hep2 cells were transfected with mock, FOXQ1-NC and FOXQ1-siRNA, and cells were seeded in 96-well plates at 4,000 cells per well. The proliferating cells were measured using a Cell Counting Kit-8 (CCK-8) assay (Beyotime Institute of Biotechnology), at 2, 4, 6 and 8 days following transfection. Cells were incubated at $37^{\circ} \mathrm{C}$ for $2 \mathrm{~h}$ following the addition of $10 \mu \mathrm{l}$ CCK- 8 to each well and the absorbance at $450 \mathrm{~nm}$ was detected using a microplate reader (MK3; Thermo Fisher Scientific, Inc., Waltham, MA, USA).

Cell cycle and apoptosis assay. For analysis of cell cycle progression and apoptosis, mock and transfected cells were fixed in $70 \%$ cold ethanol for $30 \mathrm{~min}$. After washing with cold phosphate-buffered saline (PBS) 3 times, the samples were centrifuged at $500 \mathrm{x} \mathrm{g}$ for $5 \mathrm{~min}$. The pellets were then suspended and stained with $10 \mathrm{mg} / \mathrm{l}$ propidium iodide and $100 \mathrm{mg} / 1 \mathrm{RNase}$ for $20 \mathrm{~min}$. The distribution of cells in each phase of the cell cycle and the proportion of apoptotic cells were analyzed using FACScan cytometry (Becton Dickinson, San Jose, CA, USA).

Matrigel invasion assay. Following transfection for $24 \mathrm{~h}$, $2 \times 10^{5}$ Hep2 cells were suspended in culture medium with $1 \%$ FBS and plated in the upper chamber of the Transwell plate with matrigel-coated membrane (Becton Dickinson). Cells were incubated for $36 \mathrm{~h}$, following which, cells that had not invaded through the filter were removed. Cells on the lower surface of the membrane were fixed with $4 \%$ paraformaldehyde for $15 \mathrm{~min}$, then washed with PBS and stained using hematoxylin and eosin, according to the manufacturer's 
Table I. Flow cytometry analysis of cell cycle progression and apoptosis.

\begin{tabular}{lcccc}
\hline Group & G0/G1 phase $(\%)$ & S phase $(\%)$ & G2/M phase (\%) & Apoptosis $(\%)$ \\
\hline Mock & $47.64 \pm 3.61$ & $27.55 \pm 1.54$ & $22.39 \pm 3.44$ & $2.42 \pm 0.78$ \\
FOXQ1-NC & $46.65 \pm 0.89$ & $28.64 \pm 1.85$ & $21.87 \pm 1.23$ & $2.84 \pm 2.64$ \\
FOXQ1-siRNA & $58.03 \pm 3.45^{\text {a }}$ & $23.35 \pm 0.65$ & $15.67 \pm 2.15$ & $2.95 \pm 1.65$ \\
\hline
\end{tabular}

Data are presented as the mean \pm standard deviation of three independent experiments. ${ }^{a} \mathrm{P}<0.05$, compared with the control group. FOXQ1, forkhead box Q1; NC, normal control; siRNA, small interfering RNA.

A

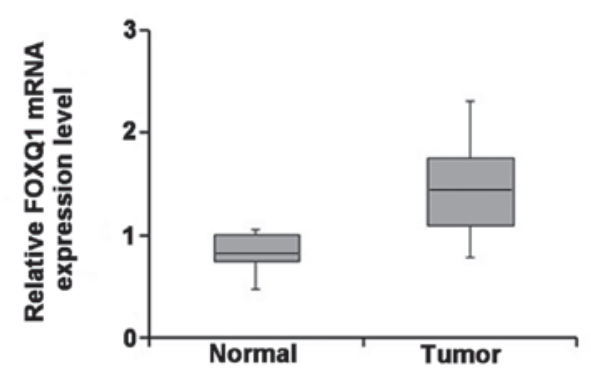

$\mathbf{B}$
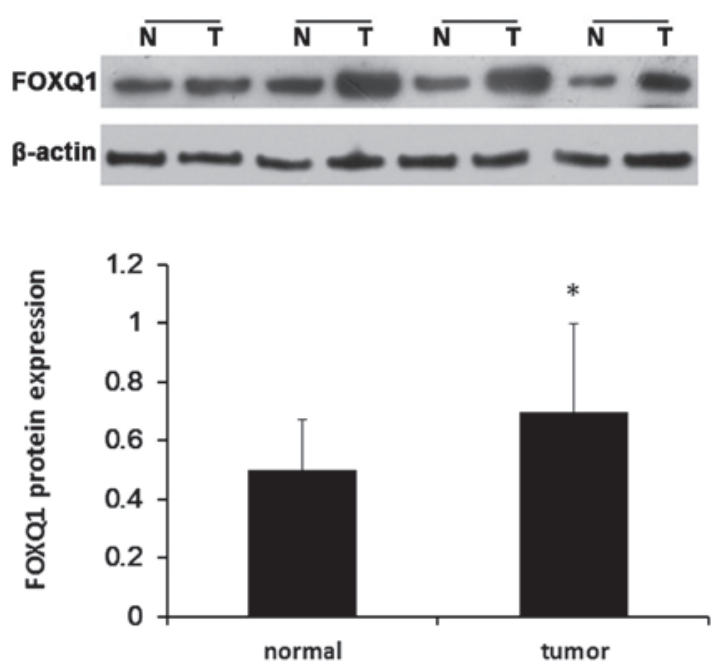

Figure 1. Expression of FOXQ1 in LSCC tissues and adjacent normal tissues. (A) FOXQ1 mRNA expression, as measured by RT-qPCR. FOXQ1 mRNA expression was detected in 30 matched LSCC tissue and adjacent normal tissue samples. Following normalization to GAPDH mRNA levels, FOXQ1 mRNA expression in LSCC tissues was shown to be higher than that in adjacent normal tissues. (B) Expression of the FOXQ1 protein in LSCC tissues and adjacent normal tissues. FOXQ1 expression in LSCC tissues (T) and adjacent normal tissues $(\mathrm{N})$ samples was detected by western blotting and $\beta$-actin was used as a control. "P<0.05 vs. normal tissues. FOXQ1, forkhead box Q1; LSCC, laryngeal squamous cell carcinoma; RT-qPCR, reverse transcription-quantitative polymerase chain reaction.

instructions. The number of cells on the membrane were counted under a microscope (CX31; Olympus Corporation, Tokyo, Japan). The number of migrated cells was expressed as the mean value of five randomly-selected fields. Each experiment was repeated three times.

Statistical analysis. All values in the present study are reported as the mean \pm standard deviation of three independent
A

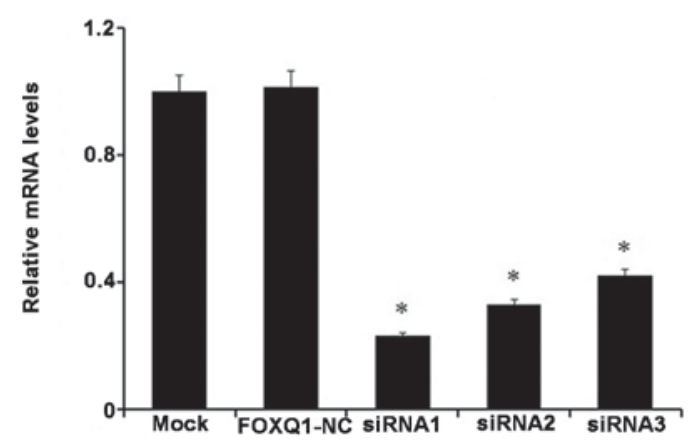

B

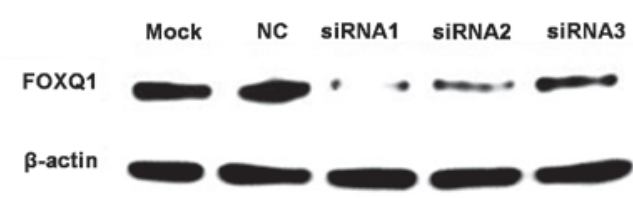

Figure 2. Knockdown of FOXQ1 mRNA and protein expression in Hep2 cells. Hep2 cells transfected with FOXQ1-siRNA or FOXQ1-NC siRNA were grown under normal culture conditions. RNA and protein were detected using RT-qPCR (A) and western blotting (B), respectively. GAPDH and $\beta$-actin were used as internal controls. ${ }^{~} \mathrm{P}<0.05$ compared with the control group. FOXQ1, forkhead box Q1; RT-qPCR, reverse transcription-quantitative polymerase chain reaction; siRNA, small interfering RNA; Mock, blank control \pm Lipofectamine 2000; NC, normal control.

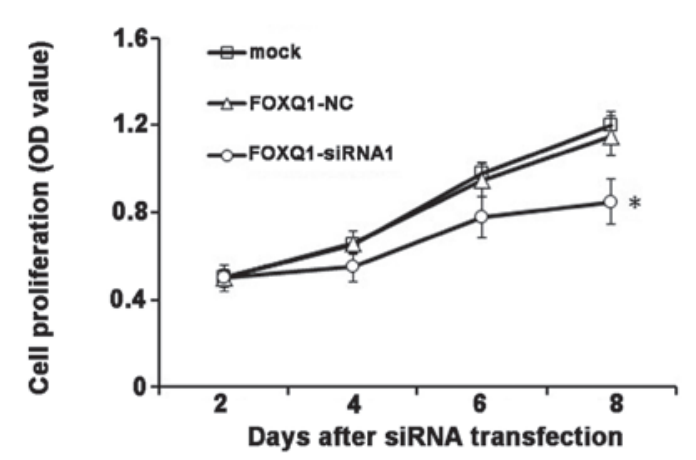

Figure 3. Downregulation of FOXQ1 expression decreased proliferation of Hep2 cells. Growth curves of Hep2 cells transfected with Hep2-NC, FOXQ1 siRNA and mock group. FOXQ1 downregulation significantly inhibited Hep2 cell growth. Each experiment was conducted in triplicate. " $\mathrm{P}<0.05$ compares with the control group. FOXQ1, forkhead box Q1; Mock, blank control \pm Lipofectamine 2000; NC, normal control; siRNA, small interfering RNA; OD, optical density.

experiments. The paired samples t-test was used to compare the expression of FOXQ1 mRNA and protein between LSCC and adjacent tissues, while one-way analysis of variance and 
A

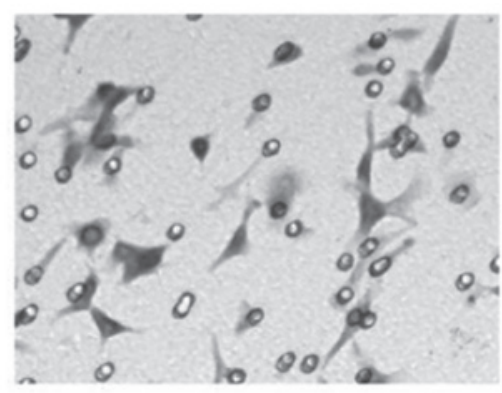

C

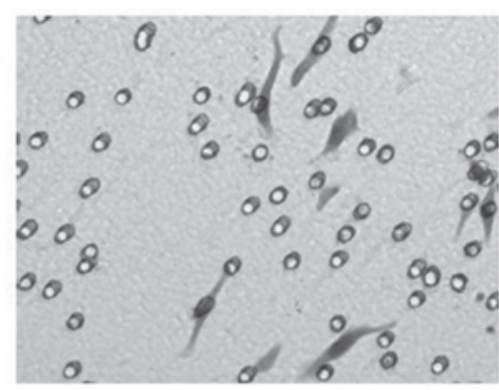

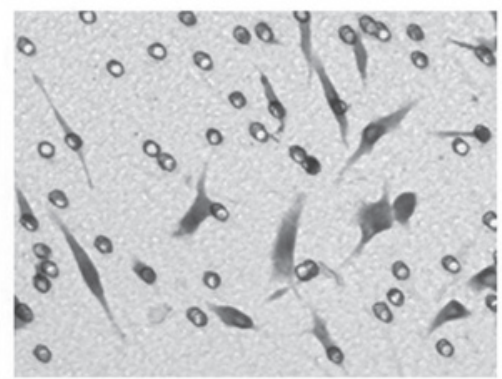

D

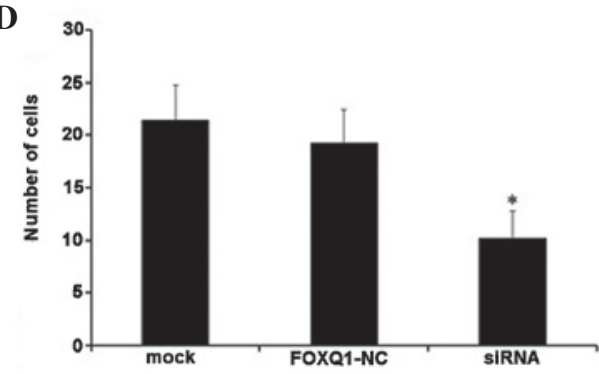

Figure 4. Invasiveness of Hep2 cells. (A) Invasiveness of Hep2 cells before treatment. (B) Invasive chart of Hep2 cells transfected with FOXQ1-NC siRNA. (C) Invasive chart of Hep2 cell transfected with FOXQ1-siRNA. (D) Number of transmembrane cells in the mock, FOXQ1-NC and FOXQ1-siRNA groups. ${ }^{*} \mathrm{P}<0.05$ compared with the control group. FOXQ1, forkhead box Q1; Mock, blank control \pm Lipofectamine 2000; NC, normal control; siRNA, small interfering RNA.

Student's t-test were used to compare values between the experimental and control groups, using SPSS 13.0 (SPSS, Inc., Chicago, IL, USA). P $<0.05$ was considered to indicate a statistically significant difference.

\section{Results}

FOXQ1 expression in LSCC tissues and adjacent normal tissues. Total RNA was extracted from 30 pairs of LSCC tissues and adjacent normal tissues and subjected to RT-qPCR in order to measure the expression of FOXQ1 mRNA. Following normalization to GAPDH, the mean expression of FOXQ1 mRNA in LSCC tissues was significantly higher than that in adjacent normal tissues $(1.54 \pm 0.66$ vs. $0.75 \pm 0.28$; $\mathrm{P}<0.05$; Fig. 1A). FOXQ1 protein expression was also measured by western blotting in the same samples in which FOXQ1 mRNA expression was measured. The results demonstrated that FOXQ1 protein expression was increased in 19 of 30 LSCC tissues ( 63\%), compared with matched adjacent normal tissues. FOXQ1 protein expression was higher in LSCC tissues than that in adjacent normal tissues (Fig. 1B; $\mathrm{P}<0.05)$. These findings were in accordance with the FOXQ1 mRNA expression data. By contrast, analysis of the association of FOXQ1 expression with characteristics, such as patient age, gender and tumor stage, revealed no significant associations between these variable (data not shown).

Inhibition of FOXQ1 following siRNA transfection in Hep2 cells. Following transfection of Hep2 cell with FOXQ1 siRNA for $72 \mathrm{~h}$, the expression of FOXQ1 mRNA and protein was detected by RT-qPCR and western blotting. Cells were also transfected with FOXQ1-NC as a negative control. The results are shown in (Fig. 2). Following transfection with FOXQ1 siRNA, Hep2 cells exhibited significant downregulation of FOXQ1 expression at the mRNA and protein levels (Fig. 2A and B; $\mathrm{P}<0.05$ ).

Downregulation of FOXQ1 expression reduces proliferation of Hep 2 cells. Cell proliferation was determined using a CCK-8 assay. The results demonstrated that downregulation of FOXQ1 in Hep2 cells resulted in a significant reduction in cellular proliferation at 4,6 and $8 \mathrm{~d}$ after transfection $(\mathrm{P}<0.05)$. This indicates that suppression of FOXQ1 correlates with decreased proliferation of Hep2 cells (Fig. 3).

Inhibition of FOXQ1 induces G0/G1 arrest, while it has no effect on apoptosis in Hep 2 cells. Flow cytometric analysis of the cell cycle demonstrated that inhibition of FOXQ1 in Hep2 cells reduced the proportion of cells in the $\mathrm{S}$ and $\mathrm{G} 2 / \mathrm{M}$ phases, and more cells were arrested in the G0/G1 phase compared with cells in the control group (Table I). Furthermore, apoptosis of FOXQ1-NC- and FOXQ1-siRNA-transfected cells was examined using flow cytometry. As shown in Table I, after 4 days, $2.42 \%$ and $2.84 \%$ of control cells and FOXQ1-NC cells were apoptotic, respectively, while $2.95 \%$ of FOXQ1-siRNA cells were apoptotic. No significant difference in the level of apoptosis in Hep2 cells was detected among these groups.

Effect of FOXQ1 silencing on cell invasion in Hep2 cell lines. The results of the matrigel invasion assay demonstrated that the number of migrating cells was significantly decreased in the FOXQ1-siRNA transfection group, compared with that in the control group. The numbers of invading cells in the mock and FOXQ1-NC groups were $21.46 \pm 3.35$ and $19.29 \pm 3.16$, respectively, which were significantly higher than the number in the FOXQ1-siRNA group (10.24 \pm 2.52 ; $\mathrm{P}<0.01$; Fig. 4). 


\section{Discussion}

FOXQ1 belongs to the forkhead transcription factor family. Previous studies have demonstrated that FOXQ1 is a downstream target of homeobox C13. Each of these may affect medullary differentiation through a common regulatory pathway $(23,24)$. A recent study reported that FOXQ1 promotes glioma cell proliferation and migration by suppressing the promoter activity of neurexin-3- $\alpha$ (NRXN3) (25). Overexpression of FOXQ1 may enhance tumor growth and tumorigenicity of colorectal cancer (19). Furthermore, overexpression of FOXQ1 is associated with a poor prognosis in non-small cell lung cancer (20) and with EMT regulation, via inhibition of E-cadherin transcription (26). To date, little is known regarding the mechanism underlying the effect of FOXQ1 on the development of human laryngeal cancer.

In the present study, FOXQ1 expression was upregulated at the mRNA and protein level in LSCC tissues, compared with adjacent normal tissues. However, no significant association was detected between FOXQ1 expression level, and gender, age or tumor stage in patients with LSCC. In order to examine whether FOXQ1 is involved in the development and progression of LSCC, RNA interference was used to reduce the expression of FOXQ1 in cultured Hep2 cells. Significant inhibition expression of FOXQ1 was observed with RT-qPCR and western blotting. In vitro suppression of Hep2 cell proliferation was analyzed, and the results demonstrated that, compared with FOXQ1-NC and mock cell groups, the proliferation of Hep2 cells was significantly inhibited following transfection with FOXQ1-siRNA.

In order to measure the effect of FOXQ1 on cell cycle progression, FACS analyses was performed, following transfection ofFOXQ1-siRNA. The results indicated that siRNA-mediated knockdown of FOXQ1, led to cell cycle arrest in the G0/G1 phase. Gao et al (27) obtained similar results and suggested that expression of FOXQ1 may affect levels of cell cycle regulators; depletion of FOXQ1 reduced the expression of cyclin E and CDK4 and increased that of the cyclin dependent kinase inhibitors (CKDIs), p27 ${ }^{\mathrm{Kip} 1}$ and $\mathrm{p} 21^{\mathrm{Cip} 1}$, which together prevented cell cycle progression.

Kaneda et al (19) and Qin et al (28) demonstrated that apoptosis was inhibited in H1299 cells overexpressing FOXQ1 and in the 7721 hepatocellular carcinoma cell line, respectively. However, in the present study, no significant difference in the level of apoptosis was detected, following suppression of FOXQ1, among the Hep2 cell groups. These findings are in accordance with those of Gao et al (27), which were conducted in the SKOV3 ovarian cancer cell line. It is therefore hypothesized that the effect of FOXQ1 on apoptosis may vary among different types of carcinoma. The mechanism underlying the influence of FOXQ1 on apoptosis requires further investigation.

The primary cause of death in almost all forms of cancer, including breast (29) and colorectal cancer (30), is cancer cell metastasis to distant organs. The initial step in metastasis is the invasion of surrounding tissues by cancer cells, and tissue invasion and metastasis are hallmarks of malignant tumors. Suppression of the pathways involved in invasion and metastasis in cancer cells may be a treatment option for patients with cancer. The results of the transwell assay in the present study, suggested that deletion of FOXQ1 in Hep2 cells transfected with siRNA may significantly reduce cell invasiveness, which further indicates that FOXQ1 is associated with the aggressiveness of LSCC cells. Sun et al (25) showed that FOXQ1 expression directly affected glioma cell migration in an NRXN3-dependent manner in vitro and in vivo. Zhu et al (31) demonstrated that suppression of FOXQ1 expression reversed the process of EMT, in association with the upregulation of E-cadherin, and that it also caused T24 bladder cancer cells to acquire an epithelial cobblestone phenotype, resulting in significantly reduced invasiveness. These results suggest that FOXQ1 is involved in tumor invasion and metastasis.

The present study demonstrated that the mRNA and protein expression of FOXQ1 was increased in LSCC tissues, compared with normal adjacent tissues. The results also showed that inhibition of FOXQ1 by transfection of siRNA into Hep2 cells significantly reduced cell growth and migration, and arrested Hep2 cells in the G0/G1 phase, in contrast to the control groups. These results indicate that FOXQ1 exhibits an oncogenic role in LSCC, which is in accordance with the results of previous studies, conducted in different types of tumors.

In conclusion, the current study has demonstrated that FOXQ1 is overexpressed in LSCC tissues, and that it may affect Hep2 cell growth, cell cycle progression and cell migration. These results suggest that FOXQ1 is a potential therapeutic target in laryngeal cancer. However, the siRNA was only transiently transfected, no long-term effects on cells were examined, and in vitro effects may differ from in vivo effects. Therefore further in vivo evaluation is required.

\section{Acknowledgements}

This study was supported by a grant from the Medicine Summit Project of Liaoning Province (grant no. 4010218).

\section{References}

1. Ragin CC, Modugno F and Gollin SM: The epidemiology and risk factors of head and neck cancer: A focus on human papillomavirus. J Dent Res 86: 104-114, 2007.

2. Jemal A, Siegel R, Ward E, Murray T, Xu J and Thun MJ: Cancer statistics, 2007. CA Cancer J Clin 57: 43-66, 2007.

3. Knab BR, Salama JK, Solanki A, Stenson KM, Cohen EE, Witt ME, Haraf DJ and Vokes EE: Functional organ preservation with definitive chemoradiotherapy for T4 laryngeal squamous cell carcinoma. Ann Oncol 19: 1650-1654, 2008.

4. Du LB, Mao WM, Chen WQ, Zhang SW, Yu CD, Zheng RS, Xia $\mathrm{QM}$ and Wang XH: Incidence and mortality of larynx cancer in China during 2003-2007. Zhonghua Liu Xing Bing Xue Za Zhi 33: 395-398 (In Chinese).

5. Cripps C, Winquist E, Devries MC, Stys-Norman D, Gilbert R; Head and Neck Cancer Disease Site Group: Epidermal growth factor receptor targeted therapy in stages III and IV head and neck cancer. Curr Oncol 17: 37-48, 2010.

6. Ji W, Guan C and Pan Z: Analysis of curative effects on laryngeal carcinoma patients in the northeast region of China. Acta Otolaryngol 128: 574-577, 2008.

7. Ozdek A, Sarac S, Akyol MU, Sungur A and Yilmaz T: c-myc and bcl-2 Expression in supraglottic squamous cell carcinoma of the larynx. Otolaryngol Head Neck Surg 131: 77-83, 2004.

8. Jain P, Kumar P, Pai VR and Parikh PM: Neoadjuvant chemotherapy or chemoradiotherapy in head and neck cancer. Indian $\mathrm{J}$ Cancer 45: 83-89, 2008

9. Bieller A, Pasche B, Frank S, Gläser B, Kunz J, Witt K and Zoll B: Isolation and characterization of the human forkhead gene FOXQ1. DNA Cell Biol 20: 555-561, 2001. 
10. Feuerborn A, Srivastava PK, KüfferS,Grandy WA,Sijmonsma TP, Gretz N, Brors B and Gröne HJ: The Forkhead factor FoxQ1 influences epithelial differentiation. J Cell Physiol 226: 710-719, 2011.

11. Korver W, Roose J and Clevers $\mathrm{H}$ : The winged-helix transcription factor Trident is expressed in cycling cells. Nucleic Acids Res 25: 1715-1719, 1997.

12. Ogaki S, Harada S, Shiraki N, Kume K and Kume S: An expression profile analysis of ES cell-derived definitive endodermal cells and Pdx1-expressing cells. BMC Dev Biol 11: 13, 2011.

13. Carlsson P and Mahlapuu M: Forkhead transcription factors: Key players in development and metabolism. Dev Biol 250: 1-23, 2002.

14. Candelario J, Chen LY, Marjoram P, Reddy S and Comai L: A filtering strategy identifies FOXQ1 as a potential effector of lamin A dysfunction. Aging (Albany NY) 4: 567-577, 2012.

15. Sizemore ST and Keri RA: The forkhead box transcription factor FOXC1 promotes breast cancer invasion by inducing matrix metalloprotease 7 (MMP7) expression. J Biol Chem 287: 24631-24640, 2012.

16. Wendling DS, Lück C, von Schweinitz D and Kappler R: Characteristic overexpression of the forkhead box transcription factor Foxf1 in Patched-associated tumors. Int J Mol Med 22: 787-792, 2008

17. Xia L, Huang W, Tian D, Zhu H, Qi X, Chen Z, Zhang Y, Hu H, Fan D, Nie Y, et al: Overexpression of forkhead box C1 promotes tumor metastasis and indicates poor prognosis in hepatocellular carcinoma. Hepatology 57: 610-624, 2013.

18. Jonsson $\mathrm{H}$ and Peng SL: Forkhead transcription factors in immunology. Cell Mol Life Sci 62: 397-409, 2005.

19. Kaneda H, Arao T, Tanaka K, Tamura D, Aomatsu K, Kudo K, Sakai K, De Velasco MA, Matsumoto K, Fujita Y, et al: FOXQ1 is overexpressed in colorectal cancer and enhances tumorigenicity and tumor growth. Cancer Res 70: 2053-2063, 2010.

20. Feng J, Zhang X, Zhu H, Wang X, Ni S and Huang J: FoxQ1 overexpression influences poor prognosis in non-small cell lung cancer, associates with the phenomenon of EMT. PLoS One 7: e39937, 2012.

21. Zhang H, Meng F, Liu G, Zhang B, Zhu J, Wu F, Ethier SP, Miller $\mathrm{F}$ and Wu G: Forkhead transcription factor foxq1 promotes epithelial-mesenchymal transition and breast cancer metastasis. Cancer Res 71: 1292-1301, 2011.
22. Sobin LH and Wittekind $\mathrm{C}$ (eds): Head and neck tumors, larynx. In: TNM Classification of Malignant Tumors. 6th edition. Wiley-Liss, New York, NY, pp52-56, 2002.

23. Potter CS, Peterson RL, Barth JL, Pruett ND, Jacobs DF, Kern MJ, Argraves WS, Sundberg JP and Awgulewitsch A: Evidence that the satin hair mutant gene Foxq1 is among multiple and functionally diverse regulatory targets for Hoxc13 during hair follicle differentiation. J Biol Chem 281: 29245-29255, 2006

24. Hong HK, Noveroske JK, Headon DJ, Liu T, Sy MS, Justice MJ and Chakravarti A: The winged helix/forkhead transcription factor Foxq1 regulates differentiation of hair in satin mice. Genesis 29: 163-171, 2001.

25. Sun HT, Cheng SX, Tu Y, Li XH and Zhang S: FoxQ1 promotes glioma cells proliferation and migration by regulating NRXN3 expression. PLoS One 8: e55693, 2013.

26. Qiao Y, Jiang X, Lee ST, Karuturi RK, Hooi SC and Yu Q: FOXQ1 regulates epithelial-mesenchymal transition in human cancers. Cancer Res 71: 3076-3086, 2011.

27. Gao M, Shih IeM and Wang TL: The role of forkhead box Q1 transcription factor in ovarian epithelial carcinomas. Int J Mol Sci 13: 13881-13893, 2012.

28. Qin J, Xu Y, Li X, Wu Y, Zhou J, Wang G and Chen L: Effects of lentiviral-mediated Foxp1 and Foxq1 RNAi on the hepatocarcinoma cell. Exp Mol Pathol 96: 1-8, 2014

29. Piccolo S, Enzo E and Montagner M: p63, Sharp1, and HIFs: Master regulators of metastasis in triple-negative breast cancer. Cancer Res 73: 4978-4981, 2013.

30. Agarwal E, Brattain MG and Chowdhury S: Cell survival and metastasis regulation by Akt signaling in colorectal cancer. Cell Signal 25: 1711-1719, 2013

31. Zhu Z, Pang Z, Xing Y, Wan F, Lan D and Wang H: Short hairpin RNA targeting FOXQ1 inhibits invasion and metastasis via the reversal of epithelial-mesenchymal transition in bladder cancer. Int J Oncol 42: 1271-1278, 2013. 